\title{
Gene expression signatures for phlegm-dampness constitution of Chinese Medicine
}

\author{
Lingru $\mathrm{Li}^{1 \dagger}$, Juan Feng ${ }^{2 \dagger}$, Haiqiang $\mathrm{Yao}^{1}$, Lan $\mathrm{Xie}^{2}$, Yu Chen ${ }^{3}$, Lingling Yang ${ }^{4}$, Shujuan $\mathrm{Hou}^{1}$, \\ Shipeng Zhao ${ }^{1}$, Ranran Sun ${ }^{1}$, Yanling $\mathrm{Wu}^{1}$, Tongtong Bai ${ }^{1}$, Yingshuai $\mathrm{Li}^{1}$, Ruoxi $\mathrm{Yu}^{1}$, Ji Wang ${ }^{1 *}$ \\ \& Qi Wang ${ }^{1 * *}$ \\ ${ }^{1}$ Center for studies in Constitution Research of Traditional Chinese Medicine, Beijing University of Chinese Medicine, Beijing 100029, China; \\ ${ }^{2}$ Medical Systems Biology Research Center, School of Medicine, Tsinghua University, Beijing 100084, China; \\ ${ }^{3}$ Nutrition Department, China-Japan Friendship Hospital, Beijing 100029, China; \\ ${ }^{4}$ Preventive Treatment of Disease Department, Zhengzhou Hospital of Traditional Chinese Medicine, Zhengzhou 450002, China
}

Received July 25, 2016; accepted November 1, 2016; published online December 5, 2016

Citation: $\quad$ Li, L., Feng, J., Yao, H., Xie, L., Chen, Y., Yang, L., Hou, S., Zhao, S., Sun, R., Wu, Y., Bai, T., Li, Y., Yu, R., Wang, J., and Wang, Q. (2017). Gene expression signatures for phlegm-dampness constitution of Chinese Medicine. Sci China Life Sci 60, 105-107. doi: 10.1007/s11427-016-0212-9

Dear Editor,

The term "individualized medicine" was first used in Biochemistry Individuality in 1956 by Williams (Williams et al., 1956). With the emergence of the post-genome era in the 21 st century, individualized precision medicine has attracted increasing attention. In traditional Chinese medicine (TCM), there is also concept of individualized medicine, which is known as the Chinese constitutional theory, proposed in the 1970s (Wang, 2012). According to this theory, the human population is divided into nine constitutions, including one balanced constitution (Normality) and eight unbalanced constitutions (Yang-deficient, Yin-deficient, Qi-deficient, Phlegm-dampness, Damp-heat, Blood stasis, Qi-stagnation, and Special intrinsic constitution). Each constitution exhibits specific physical features and psychological characteristics, and each unbalanced constitution demonstrates a specific predisposition to certain diseases and should be treated differently. The phlegm-dampness constitution is one of the eight unbalanced constitutions. Through years of clinical

$\uparrow$ Contributed equally to this work

*Correspondence author (email: doctorwang2009@126.com)

**Correspondence author (email: wangqi710@126.com) practice, we summarized the common characteristics of the phlegm-dampness constitution, including increased secretion of facial oil, dark yellow facial complexion, face and eyelid edema, copious and sticky sweat, choking sensation over the chest, copious phlegm, tuck and soft lower abdomen, drowsiness, heavy limbs, thick and greasy tongue, and slippery pulse (Wang, 2005). Persons with the phlegm-dampness constitution cannot adapt to the plum rain season and damp conditions, and they are susceptible to dampness syndrome. Epidemiologic studies show that the phlegm-dampness constitution is highly related to diabetes, hypertension, and metabolic syndromes (MS) (Wu et al., 2013).

In this study, we isolated the peripheral blood mononuclear cells (PBMCs) from 19 cases (phlegm-dampness constitution, hereinafter referred to as "T") and 17 controls (balanced constitution, normality, hereinafter referred to as " $\mathrm{N}$ "). No difference was observed between the two groups in terms of gender, age, body mass index (BMI), waist circumference, blood pressure, fasting blood glucose, triglyceride, total cholesterol, high density lipoprotein, or low density lipoprotein $(P>0.05)$ (Table $\mathrm{S} 1$ in Supporting Information).

We isolated total RNA from the PBMCs and performed the gene expression microarray. Unsupervised hierarchical 

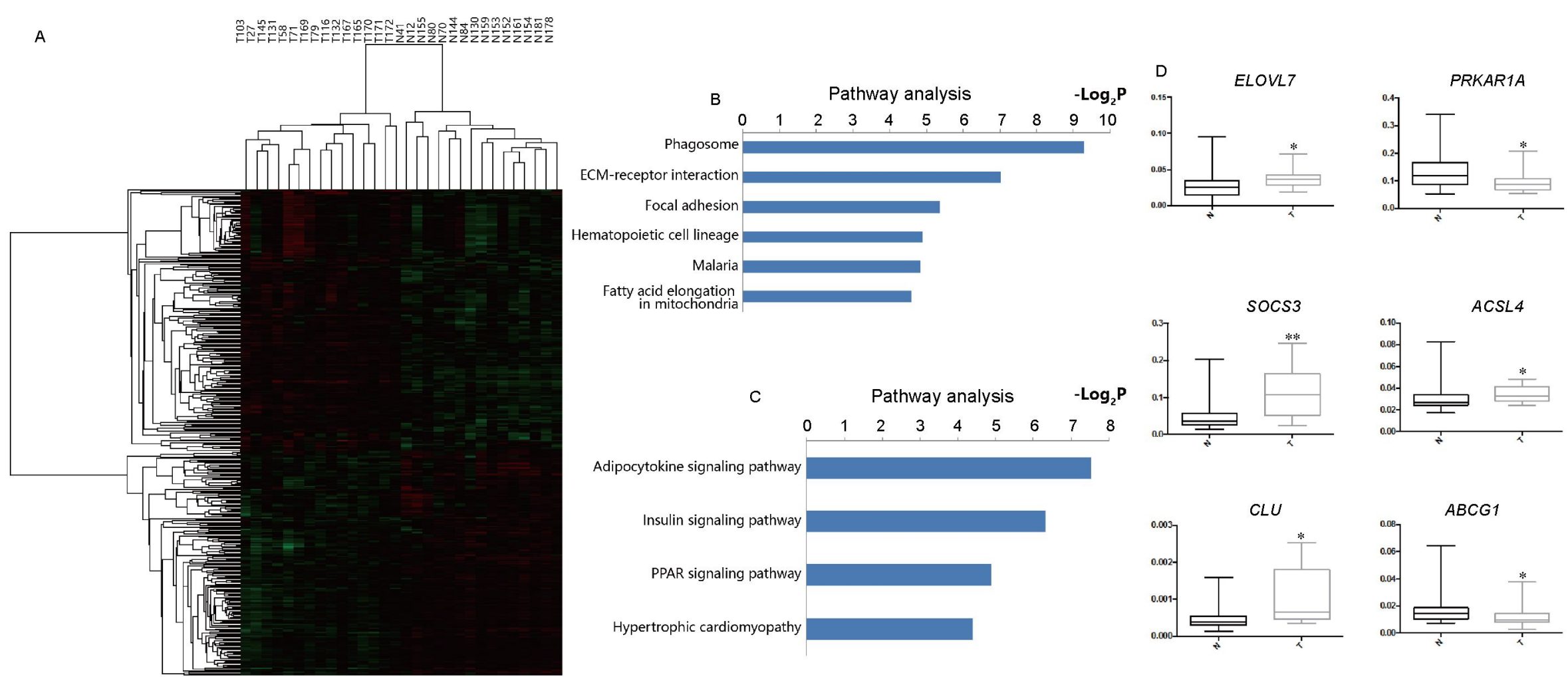

Figure 1 Expression of six metabolic disorder related genes distinguish phlegm dampness constitution from the normality group. A, Supervised hierarchical clustering of the 30 subjects based on significantly differentially expressed genes. Green to red: expression level low to high. B, $-\log 2$ of the $P$ value of the pathway analysis of the upregulated genes. C, $-\log 2$ of the $P$ value of the pathway analysis of the downregulated genes. D, Validation of differentially expressed genes via qRT-PCR. The boxplots represent the mRNA expression levels in the phlegm dampness group (in black color) versus the normality group (in gray color). The validation samples include both the original samples ( $4 \mathrm{~T}$ vs. $3 \mathrm{~N}$ ) and independent samples (15 T vs. $15 \mathrm{~N})$. The differences in the gene expression in the two groups were analyzed using

Student's $t$-tests $(*, P<0.05 ; * *, P<0.01)$ 
clustering using all probe sets was performed to discriminate the 36 subjects into two major group, which was in consistent with the grouping criteria of the TCM constitution, with the following were excluded: N134, N108, T180, T179, T175, and T168 (Figure S1A in Supporting Information). By using a multidimensional scaling algorithm, similar result was obtained that two groups were clearly distinguished from each other with a few misclassified samples (Figure S1B in Supporting Information).

The current scaling method for classifying the TCM constitution is subjective and cannot avoid cases of exaggeration or understatement of symptoms by patients. Thus, we selected the overlapping samples of scale judgment and gene expression clustering. We excluded six subjects, namely, N134, N108, T180, T179, T175, and T168. Thus, 30 subjects were included in the subsequent analysis. Based on a FDR (false discovery rate) of $<0.05$ and a fold change of $\geqslant 1.5$ cutoff, 355 differentially expressed genes were identified in the phlegmdampness constitution compared to the normality group and the phlegm-dampness and normality constitutions were distinguished well by the supervised clustering with these genes (Figure 1A). Further pathway analysis revealed that the upregulated genes were enriched in the pathways, including those in the phagosome, ECM-receptor interaction, focal adhesion, hematopoietic cell lineage, malaria, and fatty acid elongation in mitochondria while the downregulated genes were enriched in the pathways, including the adipocytokine signaling pathway, insulin signaling pathway, PPAR signaling pathway, and hypertrophic cardiomyopathy (Figure 1B and $\mathrm{C}$ ). The original samples (4 T vs. $3 \mathrm{~N}$ ) and another independent thirty subjects ( $n=15$ for T, $n=15$ for $\mathrm{N}$, the basic information was summarized in Table S2 in Supporting Information) were selected for qRT-PCR validation. No difference was observed between the two independent groups in terms of gender, age, BMI, waist circumference. Six differentially expressed genes related to different pathways or biological processes were validated. All of the six genes are associated with metabolic disorders or atherosclerosis. Among them, ELOVL7, SOCS3, ACSL4 and CLU were upregulated while $P R K A R 1 A$ and $A B C G 1$ were downregulated in the phlegm-dampness group (Figure 1D). Aberration of ELOVL 7, PRKAR1A, SOCS3, ACSL4 is indicative of the fact that people with the phlegm-dampness constitution are more susceptible with metabolic disorders and disturbance of $C L U$ and $A B C G 1$ suggests that individuals with the phlegm-dampness constitution may be at risk of atherosclerosis. This result is consistent with our previous finding, that is, the phlegmdampness constitution is highly related to diabetes, hypertension, hyperlipemia, and MS.

This work suggests that, individual difference based on Chinese constitutional theory has its biological basis. The identification of gene expression signature of the phlegm-dampness constitution enables us to further explore the molecular mechanisms of this constitution and its predisposition to related diseases.

Compliance and ethics The author(s) declare that they have no conflict of interest. The research have been approved by Medical and laboratory animal ethics committee of Beijing University of Chinese Medicine (2012BZHYLL0301). All the participants voluntarily joined this study with informed consents.

Acknowledgements This work was supported by the State Key Program of the National Natural Science Foundation of China (81030064 to Qi Wang), the National Natural Science Foundation of China (81503471 to Lingru Li, 81503471 to Ji Wang), and Beijing Natural Science Foundation (7162118 to Ji Wang). The funders had the role in study design, data collection and analysis.

Williams, R.J. (1956). Biochemical Individuality - the Basis for the Genetotrophic Concept. (New York: John Wiley \& Sons, Inc), pp. 195.

Wang, Q. (2012). Individualized medicine, health medicine, and constitutional theory in Chinese medicine. Front Med 6, 1-7.

Wang, Q. (2005). Classification and diagnosis basis of nine basic constitutions in Chinese medicine. J Beijing Univ Tradit Chin Med 28, 1-8.

Wu, X.Q., Luo,Y.Y., Xu, J.H., Ding, P., Wang, D. (2013). Investigation of traditional chinese medical constitution of type 2 diabetes mellitus patients and its relationship with insulin resistance and insulin secretion. $\mathrm{J}$ Guangzhou Univ Tradit Chin Med 30, 312-319.

Yoo, H.J., Hwang, S.Y., Hong, H.C., Choi, H.Y., Seo, J.A., Kim, S.G., Kim, N.H., Choi, D.S., Baik, S.H., and Choi, K.M. (2014). Association of metabolically abnormal but normal weight (MANW) and metabolically healthy but obese (MHO) individuals with arterial stiffness and carotid atherosclerosis. Atherosclerosis 234, 218-223.

\section{SUPPORTING INFORMATION}

Table S1 Baseline characteristics of the study subjects stratified

Table S2 Baseline characteristics of independent thirty subjects for qRT-PCR

Figure S1 A, Unsupervised hierarchical clustering of the 36 subjects. B, Analysis of the global transcriptional profiles of all the 36 samples using MDS algorithm. The red dots: normality $(\mathrm{N})$; the green dots: the phlegm dampness $(\mathrm{T})$.

The supporting information is available online at life.scichina.com and www.springerlink.com. The supporting materials are published as submitted, without typesetting or editing. The responsibility for scientific accuracy and content remains entirely with the authors. 